\title{
Pollutant of Concern: Microplastics
}

\section{Keman $S^{*}$}

Department of Environmental Health, Airlangga University, Indonesia

*Corresponding author: Professor Soedjajadi Keman, MD, Ph.D, Department of Environmental Health, Faculty of Public Health, Universitas Airlangga, Indonesia; Email: soedja_keman@fkm. unair.ac.id

\section{Editorial}

Since being discovered by John Wesley Hyatt, an American researcher, in 1968, plastic has become the prima donna for the industrial world, with total production in the world of +100 million tons per year. Plastic development is very rapid, because its ease and specialty can replace other materials such as metal and wood in helping human life. Plastics that are widely used are polyethylene (wrapping materials, plastic bags, children's toys, bottles), teflon (metal substitutes, cookware coatings), polyvinylchoride (for pipes, household appliances, paint, LPs), polytrirena (electrical insulator material, food wrapper, styrofoam, children's toys), and others. The rapid development of the synthetic polymer industry has spoiled our lives because the practicality and comfort of the products produced are characterized by a practical and comfortable life. Plastic became popular because it is lightweight, cheap, and durable. The nature of plastics that do not decompose becomes a challenge when discarded carelessly in the environment. Various impacts of plastic pollution on the environment, especially lakes, the sea and its biota have been widely reported. Some research reports reveal the other side of the ease provided by goods made of synthetic polymers in the form of toxic impacts of plastic components on human health that are still being studied to this day. Currently, the use of plastic raw materials in various commercial products continues to increase. In 2015 , plastic use reached 350 million tons. It is predicted that the number of plastic uses will increase 100-fold by 2050 .

Microplastics are formed from the breakdown of large plastic particles into small (micro) fragments that occur in nature such as ocean waters, beaches and land through biological, physical, and chemical mechanisms. This fragmentation of micro-sized plastic particles is called microplastics. The forms of microplastic particles are grouped into fibers, fragments, films, corks (foams), and beads, dominated by fibers and fragments. Karbalaei writes that microplastics are found in drinking water as many as 50 particles of per liter, 1,760-8,680 particles per kilogram honey, 550-681 particles per kilogram of table salt, 61 particles of dried fish chords, 3 particles per gram of shellfish, and 1.4-7 particles of oyster per gram. Karami study found that 16 out of 17 samples of table salt brands taken from 8 countries in North and South America, Europe, Africa, and Asia were shown to contain microplastics. This means that humans consume microplastics unconsciously and this assumption is reinforced by the discovery of all human fecal samples of microplastics. The most common types of plastic are polypropylene, polyethylene terephthalate, and polystyrene.

Microplastics with a diameter of $\leq 20 \mu$ m located in the gastroinstestinal tract will enter the blood circulation by the phagocytosis mechanism of microfold cells. After entering the blood, microplastics will be distributed throughout the body. The organs found in microplastic bioaccumulation are the liver, kidneys, spleen, heart, and brain. Microplastics in the organs of living things cause a series of biological responses such as inflammation, genotoxicity, oxidative stress, apoptosis and necrosis due to the formation of free radicals. This accelerates the process of cellular aging (apoptosis) of various organs of the body due to the occurrence of degenerative processes. Microplastic toxicity in humans requires an accumulation of doses and a certain time to be able to cause effects in the form of disease and damage to target organs. Microplastic toxic materials come from plastic monomers (plastic pellets), endogenous additives that are levied during the production process, and environmental pollutants absorbed during their stay in nature. These include phtalate, bisphenol A, polybrominate diphenyl ether and various types of heavy metals. The accumulation of microplastics in the target organs in mice triggered a free radical reaction. The discovery of microplastics in the blood is a biomarker of microplastic exposure in the environment. Furthermore, microplastics that are lipophilic will be able 
to penetrate the blood brain barrier and placenta barrier passively and undergo bioaccumulation in the target network. At the dose of toxic microplastic compounds effectively it induces the occurrence of reactive oxygen species (ROS) in the target organ cells.

If the susceptibility biomarkers consisting of superoxide dismutase (SOD), glutathione peroxidase (GPx), and catalase (CAT) are low then ROS will cause oxidative stress of the target organ cells. Damage to the target organ cells will continue at a time when antioxidant levels in the body are lower than oxidant levels. These oxidants are superoxid (O2-) hydroxyl radical $\left(\mathrm{OH}^{*}\right)$ and hydrogen peroxide (H202) formed through the Haber-Weiss reaction and Fenton reaction. The mechanism of death of target organ cells became novelty in the study explained through the path of damage to the target organ cells, namely damage to cell membranes due to superoxide anions and hydrogen peroxide, as well as damage to the DNA chromosomes of target organ cells due to radical hydroxyl translocation. Biomarkers of measurement on phospholipid membrane damage of target organs are malondialdehyde (MDA), while indicators of DNA chromoson damage are 8-oxo-7,8-dihydro2-deoxyguanosine (8-OH-dG). The presence of biomarkers of this effect indicates the occurrence of target organ cell death through necrosis and apoptosis mechanisms that can be observed in histopathological preparations in the form of necrotic bodies and apoptotic bodies. Finally, more in-depth studies are needed to reach a solid conclusion. 